\title{
Ebola Vaccine Response: An Updated Review
}

\author{
Adekunle Sanyaolu' ${ }^{1, *}$, Chuku Okorie ${ }^{2}$, Abu Fahad Abbasi $^{3}$, Stephanie Prakash ${ }^{4}$ and Aleksandra Marinkovic ${ }^{4}$ \\ ${ }^{1}$ Federal Ministry of Health, Abuja, Nigeria \\ ${ }^{2}$ Essex County College, Newark, New Jersey, USA \\ ${ }^{3}$ Loyola University Medical Center, Maywood, Illinois, USA \\ ${ }^{4}$ Saint James School of Medicine, Anguilla, British West Indies
}

*Corresponding author: Adekunle Sanyaolu, Professor Federal Ministry of Health, Abuja, Nigeria, E-mail: sanyakunle@gmail.com

Received: 18 Sep, 2020 | Accepted: 07 Oct, 2020 | Published: 14 Oct, 2020

Citation: Sanyaolu A, Okorie C, Abbasi AF, Prakash S, Marinkovic A (2020) Ebola Vaccine Response: An Updated Review. J Epidemiol Public Health Rev 5(4): dx.doi.org/10.16966/2471-8211.200

Copyright: (C) 2020 Sanyaolu A, et al. This is an open-access article distributed under the terms of the Creative Commons Attribution License, which permits unrestricted use, distribution, and reproduction in any medium, provided the original author and source are credited.

\section{Abstract}

Several studies have reported the successful use of vaccines on the Ebola virus disease (EVD). However, outbreaks were prevalent even with vaccine trials in progress; thus, we aimed to examine the response to vaccination. The response was evaluated by reviewing articles that reported the efficacy rates of the vaccine trials and provided situation reports from recent EVD outbreaks in major West African countries and the Democratic Republic of the Congo (DRC). The review findings indicated that the approved United States Food and Drug Administration (FDA) Ebola vaccine (rVZV-ZEBOV), as well as other investigational vaccines showed promising efficacy rates. It is also suggested that vaccination alone is not sufficient for proper prevention and treatment of EVD; therefore, it must be coupled with a multidisciplinary approach that requires proper training. These preventative measures may be the key to disrupting the next outbreak before it claims thousands of lives again.

Keywords: Ebola virus disease; Vaccination; Vaccines; Emergency preparedness and response

\section{Introduction}

Ebola virus disease (EVD), caused by a negative sense RNA virus, is an illness of humans and other primates from the family Filoviridae [1-4]. Infected patients generally present with symptoms within a few days to three weeks of contracting the virus [1]. These symptoms may include, but are not limited to fever, sore throat, muscle pain, and headaches; with progression to vomiting, diarrhea, rash, decreased liver and kidney functions, and internal or external bleeding [1]. The disease has a high risk of mortality within the first month following the onset of symptoms, typically due to low blood pressure resulting from fluid loss [1]. EVD patients are considered infectious and spread the virus via person-to-person contact during the symptomatic period and remain highly infective until the recovery phase; therefore, stopping the contact-transmission is possible via early detection, proper management, and vaccination [1,2].

EVD was first identified in the Democratic Republic of the Congo (DRC) in 1976 and has since evolved into a highly contagious disease with life-threatening conditions among the infected, leading to intermittent outbreaks of the virus over the past 44 years $[1,2]$. One of the most recent outbreaks of EVD caused by the strain Zaire ebolavirus started in West Africa (predominantly Guinea, Liberia, and Sierra Leone), in early 2014 [2]. This epidemic has caused vast casualty with more than 11,000 deaths and over 28,000 infected by the end of the outbreak in 2016 [2]. When the West African outbreak started, there were no vaccines approved for use on humans [3-5]. This has since changed; rVZV-ZEBOV (Ervebo), a recently licensed vaccine for prevention of EVD, was used in the 2018 to 2020 outbreak at the DRC [2-5]. Hence, this paper aimed to provide a review of the recent Ebola vaccination response.

\section{Methodology}

An electronic literature search was performed using PubMed, Google Scholar, EBSCO host, Mendeley, and Med Line Plus. The search was limited to peer-reviewed articles published between January 1, 2014, and September 16, 2020. An article was selected if it included keywords such as Ebola virus disease, vaccination, vaccines, and/or emergency preparedness and response. Articles were then reviewed and included based on the applicability to the objective of this manuscript; in addition to recreating the corresponding figures using data from the selection.

\section{Ebola Situation Report and Vaccination Response in Africa}

\section{Situation report of recent Ebola outbreaks and containments}

The Ebola outbreak of 2014 to 2016 in West Africa first started in Guinea with a young boy in December 2013 [6]. It quickly spread to the neighboring territories, Sierra Leone and Liberia, and by July 2014 all three countries were battling with the disease [6]. These countries struggled with the eradication of EVD, announcing multiple times to be "Ebola-free" only to have new cases reported [6]. The World Health Organization (WHO) removed the Public Health Emergency of International Concern (PHEIC) status on West Africa on March 
29,2016 , ending the epidemic with 28,600 cases and 11,325 mortalities $[2,6]$. Though EVD was controlled in West Africa, a cluster of cases emerged in Central Africa just a few years later. The Ministry of Health of the DRC announced a new outbreak of Ebola on August 1, 2018. It was then upgraded to a PHEIC by the Director-General of WHO on July 17,2019 , due to the spread of the virus [7]. On June 25, 2020, a public statement was issued that the EVD outbreak officially ended in the DRC [7]. There were 3,470 total EVD cases reported; with 2,287 deaths and 1,171 cases that recovered [7].

\section{Vaccination response}

Recently, there is a licensed vaccine available; in addition, other investigational vaccines are at various stages of clinical trials $[8,9]$. The preliminary results on the efficacy of the Merck recombinant vesicular stomatitis virus-Zaire Ebola virus (rVZV-ZEBOV) vaccine (Ervebo, a licensed name), have proven beneficial in the DRC outbreak and are respectively shown in figures 1 and 2 [10].

Ring vaccination using the Expanded Access/Compassionate Use protocol was recommended for the outbreak and was initiated seven days post declaration of the outbreak, on the $1^{\text {st }}$ of August 2018, following approval by the regulatory authority of DRC [10]. The ring vaccination cohort protocol in the DRC, which is based on previous experiences in West Africa, utilized a collaboration team for the ring

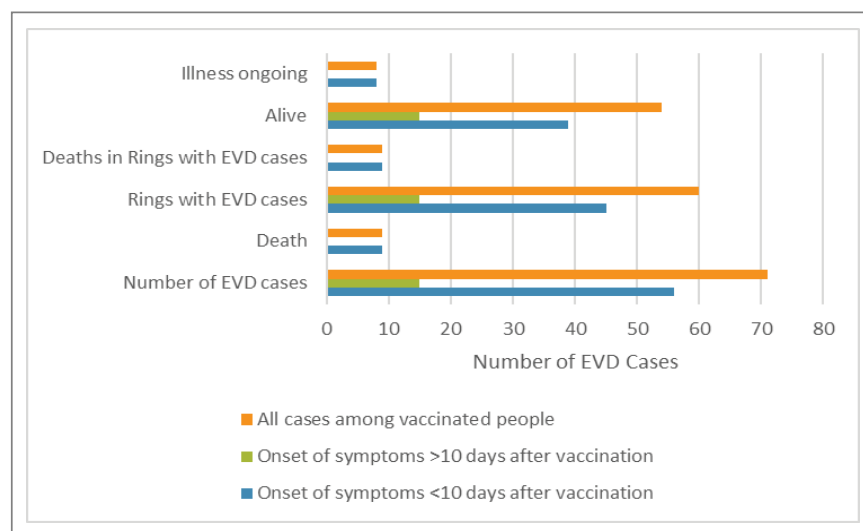

Figure 1: Distribution of confirmed EVD cases among vaccinated people at risk in the DRC with clinical outcomes.

Note: Data recreated and reported by WHO, as of April 12, 2019 [10].

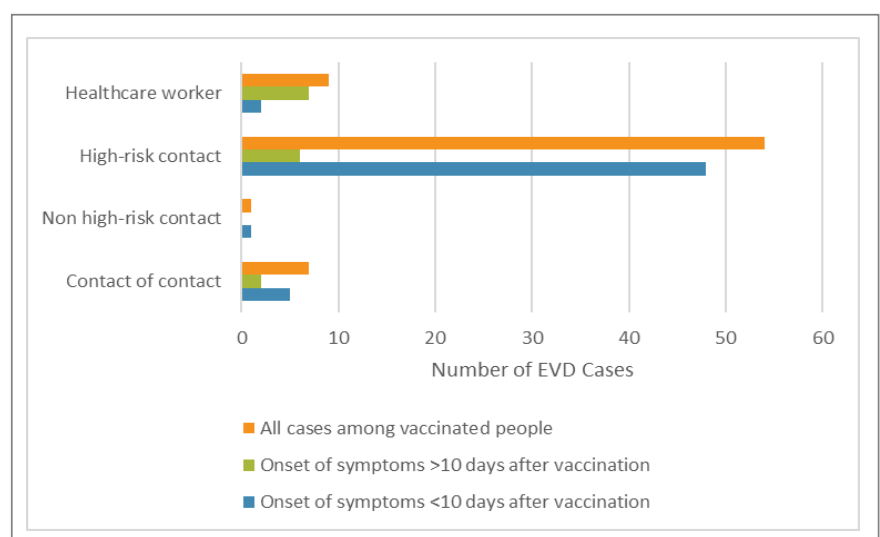

Figure 2: Distribution of confirmed EVD cases among vaccinated people in the DRC with the type of contact with the index case.

Note: Data recreated and reported by WHO, as of April 12, 2019 [10]. vaccination. The ring vaccination research teams were able to define 679 rings and enumerate contacts and contacts of contacts around 776 cases [10]. Also, 28,888 health care workers/front line workers (HCWs/FLWs) were vaccinated. Rings around 175 (18\%) of the EVD cases could not be defined. Overall, 100,754 contacts and contacts of contacts were listed and 91,492 eligible individuals who provided informed consent were vaccinated $(90.8 \%$ of all enumerated contacts and contacts of contacts were vaccinated in the defined rings) [10]. As for the characteristics of the Ebola cases, around which a ring was defined (index cases), women represented 53\% (350/658) of the index cases. The mean time from symptom onset in index cases to ring definition and vaccination was seven days. The rings had a median of 104 people. Among those at risk, $73 \%(68,279)$ included in the rings were defined as contacts of contacts. High-risk contacts accounted for $9 \%(8,123)$ of the members of the rings, while women represented $42 \%$ $(39,651)$ of the people included in the rings [10].

\section{Discussion}

During the seven months, August 2018 to March 2019, there were 951 laboratory-confirmed and probable cases of EVD in the DRC, of which 880 cases occurred among individuals who were not vaccinated and 71 occurred among the 93,965 people at risk vaccinated in 679 rings [10]. Of the 71 vaccinated cases, 15 cases ( 0 deaths) had onset of Ebola symptoms ten days or more post-vaccination [10]. This information is vital because it is the time frame after which a vaccinee is assumed to be protected by vaccination. The remaining 56 cases ( 9 deaths) had onset of symptoms less than nine days post-vaccination [10]. This duration suggests that the vaccinee was not protected or was partially protected by vaccination. Only $8.8 \%(60 / 679)$ of the vaccinated rings reported Ebola cases, and only 2.2\% (15/679) reported Ebola cases ten days or more after vaccination. Furthermore, 76\% (54/71) EVD cases occurred among the high-risk contacts who had been vaccinated. The attack rate for Ebola vaccinated individuals in the study was about $0.017 \%$, compared to $0.656 \%$ in the unvaccinated population [10]. This yields a vaccine efficacy of $97.5 \%, 95 \%$ confidence interval (95.898.5\%); therefore, indicating that vaccination has a positive effect on prevention [10].

According to WHO, Ebola outbreak control relies on multiple interventions to prevent and mitigate cases [5]. These strategies include, but are not limited to case management, surveillance and contact tracing, adequate laboratory services, safe burials, and social mobilization [5]. On August 1, 2018, the DRC declared their tenth outbreak of Ebola, which was the country's largest outbreak ever, with the number of cases surpassing 3,000 [11]. However, this is also the second-largest Ebola epidemic recorded, from the 2014 to 2016 West Africa outbreak [11]. Thus, community engagement and awareness (including vaccination) are keys to controlling outbreaks successfully [5]. Unlike the 2014 West Africa outbreak, there now exists a licensed vaccine (rVSV-ZEBOV) and the other platforms (cAd3-EBO; Ad26. ZEBOV and MVA-BN Filo) against Ebola, which are proving to be a good measure to prevent the further spread of the disease $[5,8,9,12]$. Although the recent DRC outbreak is not without its challenges, the citizens' distrust towards the EVD response is plagued by indignation due to other diseases (measles) claiming more lives and over the presence of security forces surrounding the EVD response [11].

Despite these hurdles, the new vaccine takes a novel design that incorporates a bivalent, spherical Ebola virus-like particle (VLP) with two genetically diverse glycoproteins. One of the glycoproteins encompasses the Zaire Ebola virus, while the other targets the Sudan Ebola virus [13]. The development of the live, recombinant Ebola vaccines induces immune responses that in turn protect against 
subsequent attacks [14]. This novel approach uses glycoproteins from two Ebola virus species, although researchers state that the immune response gained from vaccination might be effective against all four known pathogenic Ebola viruses, as the response to one of the glycoproteins generates cross-reactive responses against other Ebola virus species [11]. Despite data suggesting strong humoral and cellular immune responses against Ebola viruses, further research is needed to investigate vaccine effectiveness and safety, as all vaccines are still undergoing various phases of clinical trials [14]. Bache and colleagues have noted in their investigational risk to benefits study of the rVSV-ZEBOV vaccine that participants receiving the vaccine showed acceptable tolerability, safety, and satisfactory vaccineinduced immunogenicity [8]. Furthermore, the data suggests that the vaccine is appropriate in children, pregnant women, and those with immune-compromised conditions. It is their recommendation to gather additional immunogenicity data to prevent unwarranted shortcomings [8].

\section{Conclusion}

Ebola is a virus that has infiltrated multiple countries and caused thousands of casualties in recent outbreaks. It is often fatal if left untreated. Due to the alarming rate of spread of EVD, particularly on the African continent, several vaccines have been developed and have progressed through clinical trials. This review reports the efficacy of vaccination to be approximately $97.5 \%$ in the Merck recombinant vesicular stomatitis virus-Zaire Ebola virus (rVZV-ZEBOV), a recently licensed vaccine, which is a clear indication of the positive effect on the disease's prevention. The ring vaccination strategy worked due to the rapid protection after a single dose, and high coverage was achieved in the rings (91\%). Also, there is high vaccine efficacy against death, as no deaths were reported among vaccinees that developed Ebola with onset ten or more days after vaccination, and the overall case fatality rate was reduced among all vaccinees that developed Ebola. In addition to the current licensed vaccine against Zaire ebola virus species, other Ebola vaccines are advancing in clinical trials. These vaccines have comprehensive protection from different Ebola species and extended durability of protection. To prevent or mitigate any future outbreaks, vaccines must be accepted by the public, in addition to the multiple preventative measures such as proper patient care management, early diagnosis of suspected cases, outbreak tracing, isolation procedures for the infected, proper handling and disposing of contaminated materials, as well as better sanitation and burial practices.

\section{Conflict of Interest}

\section{None}

\section{References}

1. Sanyaolu S, Okorie C, Badaru O, Adler A, Boucher M, et al. (2016) Global Epidemiology of Ebola Disease: A Review. Int J Trop Dis Health 12: 1-15.

2. Wolf J, Bruno S, Eichberg M, Jannat R, Rudo S, et al. (2020) Applying lessons from the Ebola vaccine experience for SARS-CoV-2 and other epidemic pathogens. NPJ Vaccines 5: 51.

3. Marzi A, Falzarano D (2015) An updated Ebola vaccine: Immunogenic, but will it protect? Lancet 385: 2229-2230.

4. World Health Organization (2020) Ebola virus disease. WHO, Geneva, Switzerland.

5. World Health Organization (2020) Ebola virus disease: Key facts. WHO, Geneva, Switzerland.

6. Centers for Disease Control and Prevention (2019) Ebola (Ebola Virus Disease): 2014-2016 Ebola Outbreak in West Africa, CDC, United States.

7. World Health Organization (2020) Weekly epidemiological record. 95: 301-324.

8. Bache BE, Grobusch MP, Agnandji ST (2020) Safety, immunogenicity and risk-benefit analysis of rVSV- $\triangle$ G-ZEBOV-GP (V920) Ebola vaccine in phase I-III clinical trials across regions. Future Microbiol 15: 85106.

9. Saphire EO (2020) A vaccine against Ebola virus. Cell 181: 6 .

10. World Health Organization (2019) Preliminary results on the efficacy of rVSV-ZEBOV-GP Ebola vaccine using the ring vaccination strategy in the control of an Ebola outbreak in the Democratic Republic of the Congo: an example of integration of research into epidemic response. WHO, Geneva, Switzerland.

11. Medecins Sans Frontieres (2020) DRC Ebola outbreak crisis update. ecoi.net, Congo.

12. National Institute of Allergy and Infectious Disease (2020) Ebola vaccines. NIAID, United States.

13. Cincinnati Children's Hospital Medical Center (2020) New universal Ebola vaccine may fight all four virus species that infect humans. Medical Xpress.

14. Singh K, Marasini B, Chen X, Ding L, Wang JJ, et al. (2020) A Bivalent, Spherical Virus-Like Particle Vaccine Enhances Breadth of Immune Responses against Pathogenic Ebola Viruses in Rhesus Macaques. J Virol 94: e01884-e01919. 